\title{
Administration of a Probiotic Mixture Ameliorates Cisplatin- Induced Mucositis and Pica by Regulating 5-HT in Rats
}

\author{
Yuanhang Wu, ${ }^{1}$ Jianlin Wu, ${ }^{2}$ Zhikun Lin, ${ }^{3}$ Qian Wang, ${ }^{4}$ Ying Li, ${ }^{1}$ Aman Wang, Xiu Shan, \\ and Jiwei Liu ${ }^{1}{ }^{1}$ \\ ${ }^{1}$ Department of Medical Oncology, The First Affiliated Hospital, Dalian Medical University, Dalian, China \\ ${ }^{2}$ Affiliated Zhongshan Hospital of Dalian University, Dalian, China \\ ${ }^{3}$ Department of Hepatobiliary Surgery, The First Affiliated Hospital of Dalian Medical University, Dalian, China \\ ${ }^{4}$ Liaoning CapitalBio Technology Co., Ltd., Dalian, China
}

Correspondence should be addressed to Jiwei Liu; liujiwei@dmu.edu.cn

Received 22 July 2021; Revised 18 August 2021; Accepted 21 August 2021; Published 16 September 2021

Academic Editor: Tingtao Chen

Copyright (C) 2021 Yuanhang Wu et al. This is an open access article distributed under the Creative Commons Attribution License, which permits unrestricted use, distribution, and reproduction in any medium, provided the original work is properly cited.

Probiotic-based therapies have been shown to be beneficial for chemotherapy-induced mucositis. Previous research has demonstrated that a probiotic mixture (Bifidobacterium brevis, Lactobacillus acidophilus, Lactobacillus casei, and Streptococcus thermophilus) can ameliorate chemotherapy-induced mucositis and dysbiosis in rats, but the underlying mechanism has not been completely elucidated. We aimed to determine the inhibitory effects of the probiotic mixture on cisplatin-induced mucositis and pica and the underlying mechanism, focusing on the levels of 5-hydroxytryptamine (5-HT, serotonin) regulated by the gut microbiota. A rat model of mucositis and pica was established by daily intraperitoneal injection of cisplatin $(6 \mathrm{mg} / \mathrm{kg})$ for 3 days. In the probiotic+cisplatin group, predaily intragastric injection of the probiotic mixture $\left(1 \times 10^{9} \mathrm{CFU} / \mathrm{kg}\right.$ BW) was administrated for 1 week before cisplatin injection. This was then followed by further daily probiotic injections for 6 days. Histopathology, pro-/anti-inflammatory cytokines, oxidative status, and 5-HT levels were assessed on days 3 and 6 . The structure of the gut microbiota was analyzed by $16 \mathrm{~S}$ rRNA gene sequencing and quantitative PCR. Additionally, 5-HT levels in enterochromaffin (EC) cells (RIN-14B cell line) treated with cisplatin and/or various probiotic bacteria were also determined. The probiotic mixture significantly attenuated kaolin consumption, inflammation, oxidative stress, and the increase in 5-HT concentrations in rats with cisplatin-induced intestinal mucositis and pica. Cisplatin markedly increased the relative abundances of Enterobacteriaceae_other, Blautia, Clostridiaceae_other, and members of Clostridium clusters IV and XIVa. These levels were significantly restored by the probiotic mixture. Importantly, most of the genera increased by cisplatin were significantly positively correlated with colonic 5-HT. Furthermore, in vitro, the probiotic mixture had direct inhibitory effects on the 5-HT secretion by EC cells. The probiotic mixture protects against cisplatin-induced intestine injury, exhibiting both anti-inflammatory and antiemetic properties. These results were closely related to the reestablishment of intestinal microbiota ecology and normalization of the dysbiosis-driven 5-HT overproduction.

\section{Introduction}

Cisplatin is a chemotherapy agent used for the first-line treatment of the majority of cancer patients [1]. Although clinical trials have shown effectiveness, unfavorable cytotoxic side effects are a huge hurdle that impedes the clinical application of otherwise beneficial cisplatin-based treatments [2-4]. Intestinal mucositis, a serious chemotherapy-induced side effect, is characterized by local accumulation of inflammatory cells [5], cell loss in the epithelial barrier [6], increased oxidative stress [7], and reduced gastrointestinal digestive enzyme activities [8]. Clinical manifestations include nausea, vomiting, ulceration, bloating, anorexia, constipation, severe diarrhea, and subsequent weight loss [9-11], causing potentially life-threatening complications. Improved management of cisplatin-induced mucositis and nausea/vomiting may help avoid reductions in and discontinuation of chemotherapy among patients. 
Advances during the past three decades have elucidated some of the mechanisms by which chemotherapeutic agents induce side effects. Among these mechanisms, the effect of the neurotransmitter 5-hydroxytryptamine (5-HT, serotonin) plays an important part in chemotherapy-induced mucositis [9]. Cisplatin treatment may cause free radical generation, leading to localized exocytotic release of 5-HT from the enterochromaffin (EC) cells [10]. Thereafter, 5-HT interacts with 5 -HT3 receptors on vagal afferent terminals in the wall of the bowel and transmits the stimulus to the brain, resulting in emesis [10]. 5-HT also regulates interstitial cells of Cajal (ICCs) in the gastrointestinal (GI) tract [12] and aquaporin 3 (AQP3) expression in the colon [13], which is closely related to constipation and diarrhea. In addition, increased intestinal 5-HT level coincides with deregulation of the mucosal immune system, which in turn exacerbates damage [14]. Currently, most drugs used as prophylaxis for chemotherapy-induced side effects belong to the classes of 5-HT3 receptor antagonists [15]. As a result, the quality of life for cancer patients has improved to a certain degree. However, these antagonists can also cause central nervous system (CNS) side effects, such as headaches, dizziness, and abnormal vision [16]. Therefore, adjuvant therapy targeting the biosynthesis of 5-HT by EC cells is a promising way to ameliorate chemotherapy-induced mucositis.

More than $90 \%$ of the total 5-HT in the human body is synthesized, stored, and released by EC cells in the intestinal mucosa [17]. 5-HT biosynthesis by EC cells was recently found to be modulated by human gut bacteria, such as Clostridium spp. [18]. This suggests a critical interaction between the gut microbiota and chemotherapy-induced GI complications. Disturbance of the commensal microbial structure, so-called dysbiosis, will inevitably influence EC cells and eventually affect the 5-HT-mediated stimulation of the GI tract and brain [19]. In recent years, several studies have explored gut dysbiosis in patients taking cisplatinbased chemotherapy [8, 20-22]. However, few studies have systematically evaluated the possible correlation between gut dysbiosis and 5-HT-mediated GI mucositis. In addition, whether reduction of chemotherapy-induced dysbiosis by probiotics can ameliorate the 5-HT overproduction by EC cells remains unclear.

Although rats do not vomit [23], they exhibit pica behavior (eating of nonnutritive substances such as kaolin) in response to a variety of emetogenic stimuli, which can be used as a proxy variable for nausea and vomiting in rat models $[24,25]$. In this study, we administered a probiotic mixture (Bifidobacterium brevis DM8310, Lactobacillus acidophilus DM8302, Lactobacillus casei DM8121, and Streptococcus thermophilus DM8309) to rats with cisplatin-induced mucosal damage and pica. We aimed to investigate its effects on inflammation, oxidative stress, gut microbiota modification, and the levels of serum and colonic 5-HT in the rats. Our results showed that the probiotic mixture ameliorated cisplatin-induced mucositis and pica in rats and normalized the dysbiosis-driven 5-HT overproduction. These effects may be closely related to the effects of the probiotic mixture on gut dysbiosis in the cisplatin-treated rats.

\section{Materials and Methods}

2.1. Bacterial Strains and Drugs. A probiotic mixture, including the following strains: Bifidobacterium brevis DM8310, Lactobacillus acidophilus DM8302, Lactobacillus casei DM8121, and Streptococcus thermophilus DM8309, was provided by Dalian Medical University and prepared as described in a previous study [26]. Briefly, the strains were cultured separately on solid media under anaerobic condition, at $37^{\circ} \mathrm{C}$ for $24 \mathrm{~h}$. After that, single colonies were picked and inoculated into MRS liquid medium and cultured for 24 hours. The bacteria were then mixed by centrifugation at $5,000 \times g, 4^{\circ} \mathrm{C}$ for $2 \mathrm{~min}$, and resuspended in $2 \mathrm{~mL}$ PBS before administration to rats. Cisplatin was obtained from Qilu Pharmaceutical Co., Ltd. (China) and dissolved in saline. Kaolin, acquired from China Pharmaceutical Chemical Reagents Co., Ltd. (China), was prepared and processed as previously described [21].

2.2. Animal Study. Sprague-Dawley (SD) male rats aged 8 weeks old and weighing 180 200 g each were supplied by the Specific Pathogen Free animal centre of Dalian Medical University and were kept on a $12 \mathrm{~h}$ light/dark cycle with access to food and water ad libitum in a temperaturecontrolled room $\left(25^{\circ} \mathrm{C}\right)$. The kaolin pellets were introduced into the rats 6 days prior to cisplatin injection. Most of the rats stopped taking Kaolin on the third day, and those that were still interested in kaolin were excluded. The remaining rats were randomly divided into 3 experimental groups ( $n=10$ each), and toxicity was induced by daily intraperitoneal injection of cisplatin to rats with the dosage of $6 \mathrm{mg} / \mathrm{kg}$ for 3 days. The rats in the control group (Con) were subjected to saline $(0.9 \% \mathrm{NaCl})$ injection every day and saline daily by gavage. The cisplatin group (Cis-p) received saline daily by gavage. The probiotic+cisplatin group (PM) was given cisplatin injection daily for 3 days and administration of probiotic mixture $\left(1 \times 10^{9} \mathrm{CFU} / \mathrm{kg}\right.$-BW) daily for 6 days. Probiotic mixture was administered one week before cisplatin injection. The general conditions of rats were examined every day, including activities, fur, appetite, breath, and stool. The body weight and consumption of kaolin were recorded every $24 \mathrm{~h}$ until the rats were sacrificed after anesthesia with sodium pentobarbital on the assigned day of each experiment. The small intestine (corresponding to the jejunum and ileum, resp.) and colon were removed. All animal work was performed according to the laboratory's animal ethics guidelines, and protocols were approved by the Dalian Medical University Institutional Animal Care and Use Committee (SYXK [Liao] 2017-0003).

\subsection{Intestinal Histology and Immunofluorescence Staining.} The colon of the rat was cut into distal, medial, and proximal sections, and $1 \mathrm{~cm}$ of different sections was fixed in $4 \%$ paraformaldehyde overnight at $4^{\circ} \mathrm{C}$ and then embedded in paraffin, and $4 \mu \mathrm{m}$ thick sections were prepared. For histological analysis, sections were stained with hematoxylin/eosin (HE) and analyzed. The severity of colon damage was semiquantitatively scored according to a histological scoring scale previously described [27]. For double-label immunofluorescence immunohistochemistry, slides were blocked with 
$10 \%$ NDS containing $0.3 \%$ Triton $\mathrm{X}-100$ at $4^{\circ} \mathrm{C}$ for $2 \mathrm{~h}$ and incubated with rabbit anti-mouse $\operatorname{CgA}(1: 500$, Abcam) in combination with a rat anti-mouse 5-HT (1:50; Abcam) antibody at $20^{\circ} \mathrm{C}$. After washing, the sections were incubated with a mixture of Alexa Fluor 488-conjugated donkey antirabbit IgG and Alexa Fluor 594-conjugated donkey anti-rat IgG (Invitrogen, Rockford, IL, USA; 1:1,000) secondary antibodies at $20^{\circ} \mathrm{C}$ for $3 \mathrm{~h}$ while protected from light. The sections were then mounted on glass slides and finally embedded with Fluoromount/Plus (K048, Diagonostic Biosystems, Pleasanton, CA, USA) after drying at room temperature for $30 \mathrm{~min}$.

2.4. ELISA. The levels of lipopolysaccharides (LPS, USCN, USA), malondialdehyde (MDA, Nanjing Jiancheng Bioengineering Institute, China), serotonin, and cytokines (USCN, USA) including TNF- $\alpha$, IL-6, and IL-10 in sera and/or supernatant of tissue homogenates were detected by ELISA according to the manufacturer's instructions. Readings from tissue samples were normalized to total protein content as detected by the BCA assay (Thermo Pierce). Data that compiled across multiple experiments are expressed as concentrations normalized to controls within each experiment.

2.5. The Quantitative Real-Time PCR ( $q P C R$ ) Detection. The extraction of total RNA and the synthesization of the complementary DNA (cDNA) were performed as previously described [28]. For detection of rat genes, amplification was performed in triplicates in 384 well plates (QuantStudio 6 Flex Real-Time PCR System) using ChamQ Universal SYBR qPCR Master Mix (Vazyme). The expression levels were calculated using the comparative $2^{-\triangle \Delta c t}$ method. For detection of fecal bacterial groups of rats, amplification and detection were performed as previously described [26]. Bacterial quantity was expressed as $\log 10$ bacteria/g of fecal content. qPCR primers are listed in Table 1.

2.6. $16 S$ rRNA Gene Sequencing and Analysis. The fecal metagenomic DNA of rats was extracted using the QIAamp DNA Stool Mini Kit (Qiagen, Hilden, Germany). The primer pair 520F/802R was used to amplify the V3-V4 hypervariable region of the bacterial $16 \mathrm{~S}$ rDNA from bacteria in rat feces of six groups $(n=4-5$ each). HiSeq sequencing and data analysis were subsequently performed using a method described previously [29]. Operational taxonomic units (OTUs) present in $50 \%$ or more of the fecal samples were identified as core OTUs. Principal component analysis (PCA) was then conducted according to the distance matrices to analyze the diversity between groups.

2.7. RIN-14B In Vitro Culture Experiments. RIN-14B cells were purchased from ATCC (ATCC No. CCL 89) and seeded into 24 -well plates at a density of $2 \times 10^{5}$ cells $/ 0.5 \mathrm{~mL}$ RPMI1640 medium (Invitrogen-Japan) with 10\% FCS (Thermo Fischer Scientific), 1\% penicillin and streptomycin (Thermo Fisher Scientific) at $37^{\circ} \mathrm{C}$, and $5 \% \mathrm{CO}_{2} .1 \mathrm{~mL}$ of the bacteria culture broths $(\mathrm{OD} 600=1.5)$ was centrifuged at $2500 \mathrm{rpm}$ for 5 minutes, and supernatants were filtered through $0.2 \mu \mathrm{m}$ pore syringe filters. Cultured RIN-14B cells were incubated with different bacterial filtrates for $1 \mathrm{~h}$ at $37^{\circ} \mathrm{C}$. For cisplatin treatment, cells were incubated with cisplatin $(1 \mu \mathrm{g} / \mathrm{mL})$, passed through $0.2 \mu \mathrm{m}$ pore syringe filters, while for the cisplatin combined with PM treatment, cells were incubated with cisplatin $(1 \mu \mathrm{g} / \mathrm{mL})$ and $1 \mathrm{~mL}$ probiotic mixture. After incubation, the supernatant was collected, which was then centrifuged $6000 \times g$ for $5 \mathrm{~min}$ and frozen for downstream 5-HT analysis.

2.8. Statistical Analysis. All quantitative data were expressed as the mean \pm standard error of the mean (SEM). Statistical analyses were performed using two-tailed, unpaired Student's $t$-test for comparisons of two groups when data obey normal distribution and even variance and using one-way ANOVA followed by the Kruskal-Wallis rank sum test for multigroup comparisons. Pearson's correlation test was used to analyze the correlation between the differential microbes and colon 5-HT level. Significance was set at ${ }^{*} p<0.05,{ }^{* *} p<0.01,{ }^{* * *} p<0.001$, and ${ }^{* * * *} p<0.0001$.

\section{Results}

3.1. Probiotic Mixture Ameliorates Cisplatin-Induced Pica and Mucositis in Rats. We established a rat model of pica and intestinal mucositis by intraperitoneally injecting cisplatin. The experiment design is shown in Figure 1(a). During the $6 \mathrm{~d}$ experiment, one rat died after cisplatin treatment in the Cis-p group on day 5 (Figure 1(b)). No other rats died. The body weight and kaolin consumption level (indicating the degree of pica) were measured. The body weight of rats in the Cis-p group decreased $(p<0.0001$ vs. the Con group, Figure $1(\mathrm{c})$ ). The probiotic mixture ameliorated the decreased body weight to a certain extent ( $p=0.0002$ vs. the Cis-p group, Figure 1(c)). Before the first injection of cisplatin, the rats in the different experimental groups consumed a similar amount of kaolin $(p>0.05$ between each pair of groups). After injection of cisplatin, the kaolin consumption was significantly increased in the cisplatin-only rats compared to the control rats. Kaolin consumption in the PM group decreased to a certain extent, indicating that the probiotic mixture can ameliorate cisplatin-induced pica in rats (Figure $1(\mathrm{~d})$ ).

\subsection{Probiotic Mixture Improves the Intestinal Barrier} Function in Cisplatin-Induced Mucositis. Morphologic observation of the colon tissue of rats after cisplatin treatment revealed severe mucosal lesions with villous atrophy, irregular arrangement and architecture of the epithelial cell, and crypt disruption in the colon mucosa compared to the control rats. Compared to the cisplatin-only rats, the probiotic mixture protected the colon mucosa from cisplatininduced injury by improving these factors (Figure 2(a)). The histological score of the colon in rats treated with cisplatin was higher compared with control and the probiotic mixture administrated rats (day 3: Con vs. Cis-p, $p<0.0001$; Cis-p vs. PM, $p<0.0001$; day 6: Con vs. Cis-p, $p<0.0001$; Cis-p vs PM, $p=0.0082$; Figure 2(b)). A significantly elevated serum lipopolysaccharide (LPS) level, which indicates gut permeability, was found in the cisplatinonly rats $(p=0.0003)$, and the probiotic mixture significantly 
TABLE 1: List of PCR primers used for qPCR detection of gene expression and bacterial groups.

\begin{tabular}{|c|c|c|c|}
\hline Gene/bacterial group & Sequences $\left(5^{\prime}-3^{\prime}\right)$ of primers & Size (bp) & Reference \\
\hline MUC-2 & $\begin{array}{l}\text { Forward: GGCTATGGCAGACTTTGT } \\
\text { Reverse: GCATTTGCGAGTTATCAG }\end{array}$ & $262 \mathrm{bp}$ & This study \\
\hline TPH1 & $\begin{array}{l}\text { Forward: ACCATCTTCCGAGAGCTGAA } \\
\text { Reverse: GATGGAAAACCCTGTGCGTT }\end{array}$ & $162 \mathrm{bp}$ & [71] \\
\hline TPH2 & $\begin{array}{l}\text { Forward: ATCCCAAGTTTGCTCAGTTTT } \\
\text { Reverse: GATGGACGAAAGTAACCCTG }\end{array}$ & $167 \mathrm{bp}$ & [71] \\
\hline SERT & $\begin{array}{l}\text { Forward: AACTGGCAGAAACTCTTGGA } \\
\text { Reverse: GAAGATGACGAAGCCAGAGA }\end{array}$ & $195 \mathrm{bp}$ & [71] \\
\hline$\beta$-Actin & $\begin{array}{l}\text { Forward: TGGCACCACACTTTCTACAAT } \\
\text { Reverse: GGTACGACCAGAGGCATACA }\end{array}$ & $189 \mathrm{bp}$ & This study \\
\hline Clostridium cluster XIVa & $\begin{array}{l}\text { Forward: AAATGGACGGTACCTGACTAA } \\
\text { Reverse: CTTTGAGTTTCATTCTTGCGAA }\end{array}$ & $441 \mathrm{bp}$ & [72] \\
\hline Clostridium cluster IV & $\begin{array}{l}\text { Forward: TTACTGGGTGTAAAGGG } \\
\text { Reverse: CTTCCTCCGTTTTGTCAA }\end{array}$ & $580 \mathrm{bp}$ & [73] \\
\hline
\end{tabular}
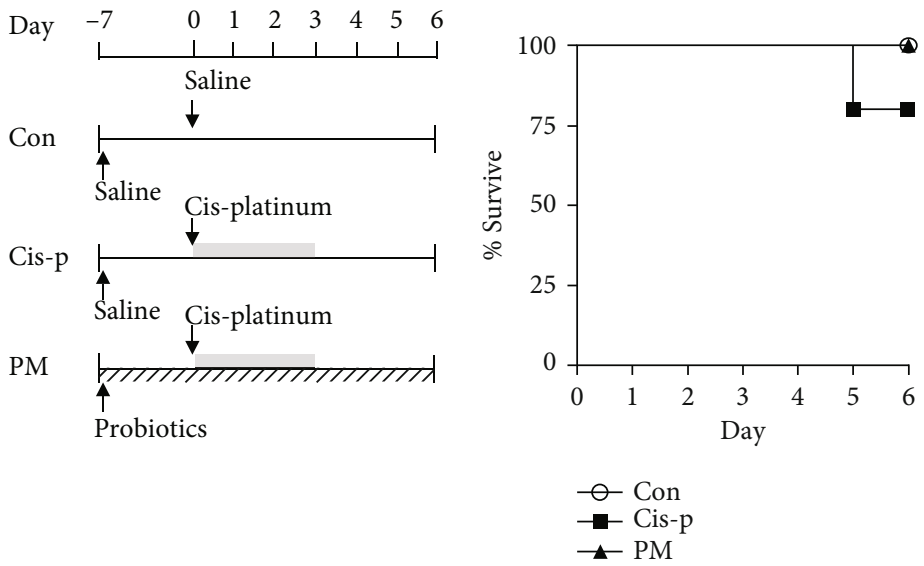

(a)

(b)
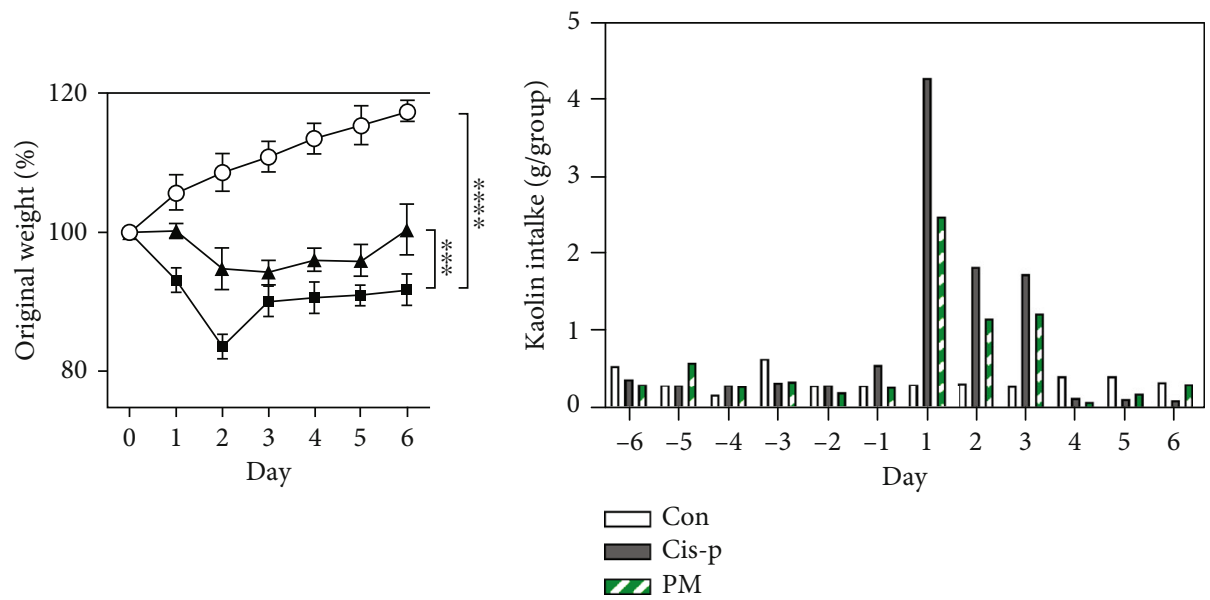

(c)

(d)

FIGURE 1: Effects of probiotic mixture on cisplatin-induced mucositis and pica in rats $(n=5)$ : (a) experimental setup, (b) survival rate, (c) body weight, and (d) kaolin consumption. Con: normal control group; Cis-p: cisplatin-treated model group; PM: probiotic mixture- and cisplatin-treated group. 

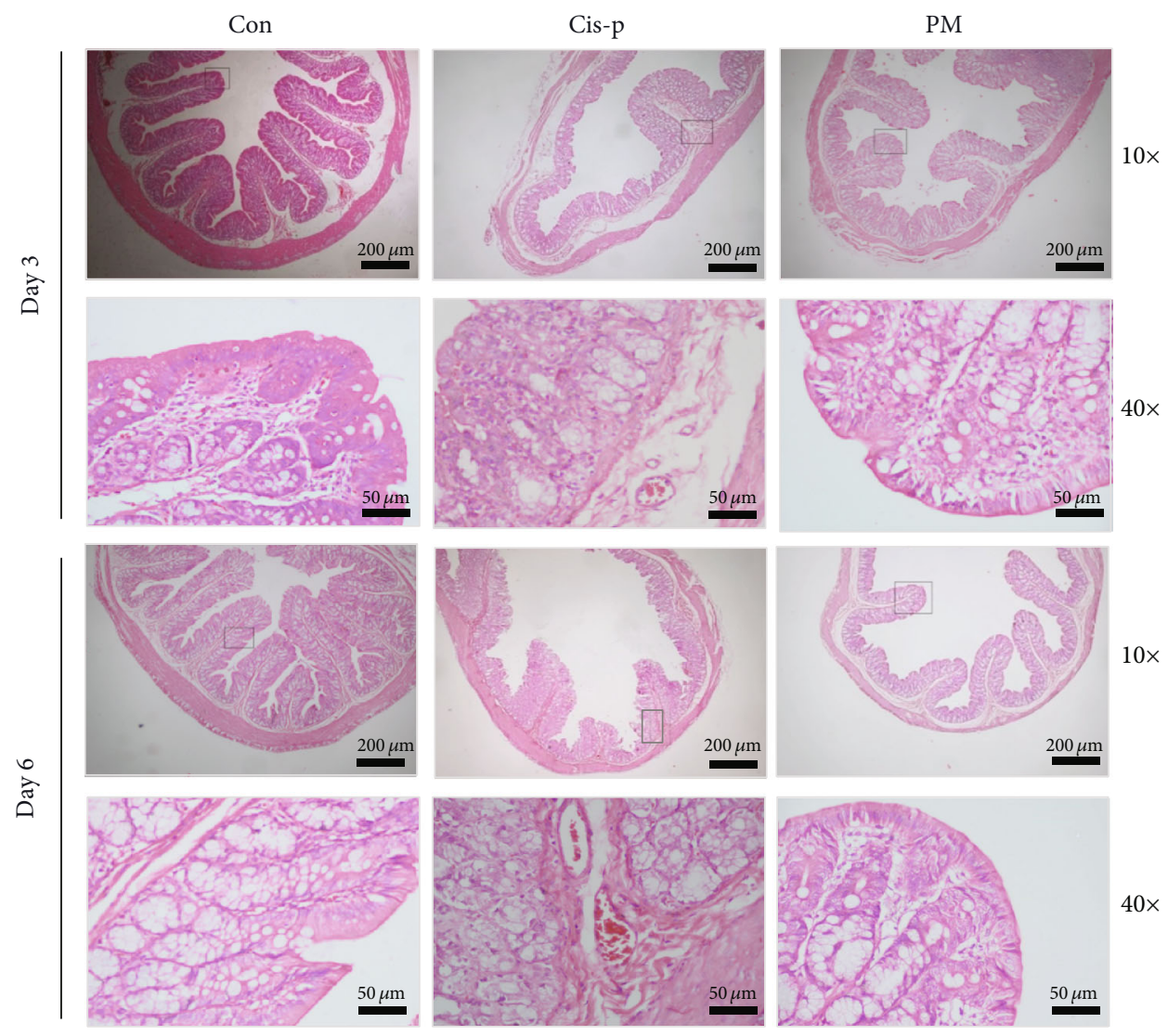

$0 \mu \mathrm{m}$
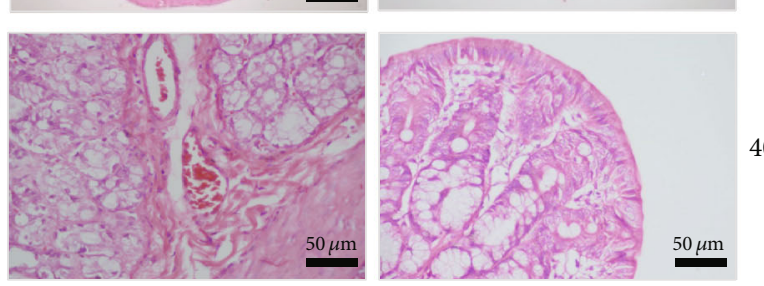

(a)
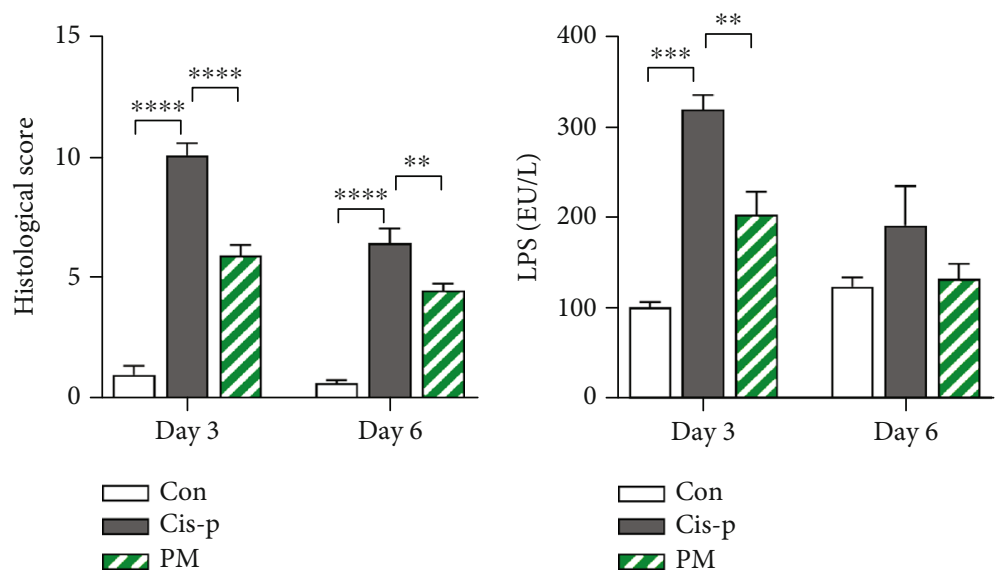

(b)

(c)

Figure 2: Continued. 

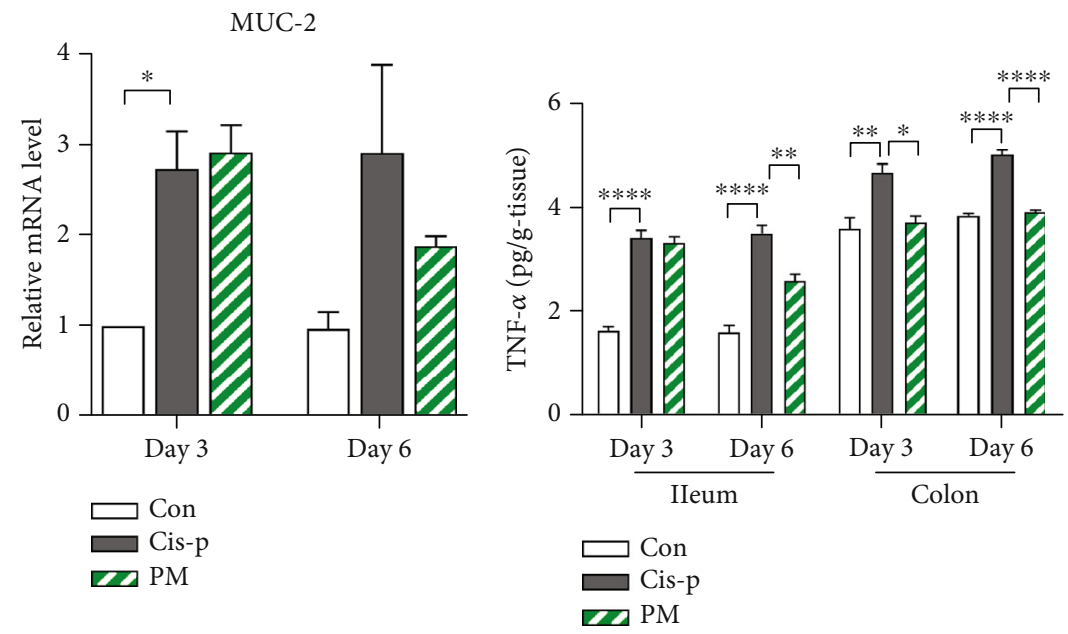

(d)

(e)

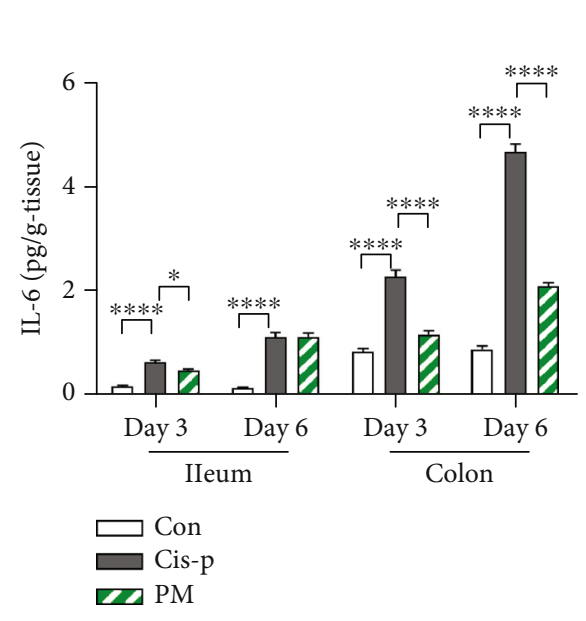

(f)

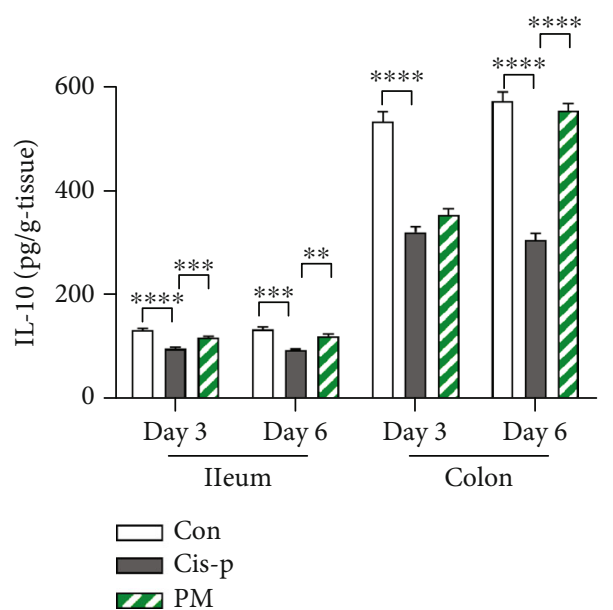

(g)

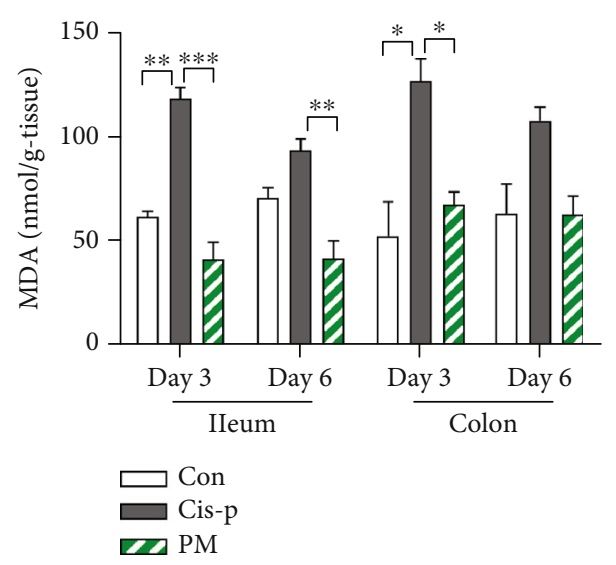

(h)

FIGURE 2: Effects of probiotic mixture on the intestinal barrier function in cisplatin-induced mucositis. (a) H\&E staining of rat colon sections. (b) Histopathological analysis of H\&E-stained sections. (c) Serum LPS were detected by ELISA. (d) Relative mRNA expression of mucin-2 (MUC-2) in colon tissues of rats, detected by quantitative PCR. Levels of tumor necrosis factor- (TNF-) $\alpha$ (e), interleukin(IL-) 6 (f), IL-10 (g), and malondialdehyde (MDA) (h) in the ileum and colon. Data are expressed as mean \pm SEM $(n=3-5):{ }^{*} p<0.05$, ${ }^{* *} p<0.01,{ }^{* * *} p<0.001$, and ${ }^{* * * *} p<0.0001$. 
inhibited this increase $(p=0.0088$; Figure 2(c)). Mucin-2 (MUC-2) is the key constituent of the mucosal barrier that protects the mucosal epithelial layer. The mRNA expression levels of MUC-2 in the colon were significantly increased in the Cis-p group on day 3 ( $p=0.0142$; Figure $2(d)$ ) and nonsignificantly decreased in the PM group on day 6 . Additionally, compared to the Cis-p group, the probiotic mixture led to further beneficial effects on both days 3 and 6 , such as reducing the elevated intestinal level of inflammatory tumor necrosis factor- (TNF-) $\alpha$ (ileum: $p=0.0025$ [day 6]; colon: $p=0.0107$ [day3]; $p<0.0001$ [day 6]; Figure 2(e)) and interleukin- (IL-) 6 (ileum: $p=0.0136$ [day 3]; colon: $p<0.0001$ [day3]; $p<0.0001$ [day 6]; Figure 2(f)) and promoting the secretion of IL-10 (ileum: $p=0.0008$ [day 3]; $p=0.006$ [day 6]; colon: $p<0.0001$ [day 6]; Figure $2(\mathrm{~g})$ ). These results suggested that the probiotic mixture has an anti-inflammatory effect on cisplatin-induced intestinal mucositis in rats. Malondialdehyde (MDA), a product of lipid peroxidation, is an oxidative stress biomarker. Cisplatin increased the MDA level in intestinal tissues on both days 3 and 6 , while the probiotic mixture significantly decreased the MDA concentration (ileum: $p=0.0008$ [day 3]; $p=0.006$ [day 6]; colon: $p=0.0001$ [day 3]; Figure 2(h)). Together, these data indicated that the probiotic mixture had significant effects on the intestinal barrier functions, including the mechanical, immune, and chemical barrier functions.

3.3. Probiotic Mixture Reduces Serum and Colonic 5-HT Overproduction. As cisplatin-induced emesis is associated with an increase in 5-HT concentration, produced by EC cells, we conducted immunofluorescent staining of rat colon tissues in the three groups and also determined the colonic and serum levels of 5-HT. Double staining of chromogranin A (GI endocrine cell marker) and 5-HT showed that cisplatin-induced increases in 5-HT were localized to the colonic EC cells (Figure 3(a)). The levels of 5-HT in the colon and serum of the cisplatin-only rats were significantly increased in comparison to the control group (colon: $p=0.0171$ [day 3]; $p=0.0327$ [day 6]; serum: $p=0.0056$ [day 3]; $p=0.0277$ [day 6]; Figures 3(b) and 3(c)), and the probiotic mixture reduced the cisplatin-induced increases in colonic and serum levels of 5-HT, with significant differences on both days 3 and 6 (colon: $p=0.018$ [day 3]; $p=0.0148$ [day 6]; serum: $\mathrm{P}=0.0012$ [day 3]; $P=0.0238$ [day 6]; Figures 3(b) and 3(c)).

To investigate whether the inhibitory effects of the probiotic mixture on 5-HT levels in rats are due to the regulation of 5-HT synthesis or transport, we assessed the mRNA expression of tryptophan hydroxylases (TPH1 and TPH2) and the serotonin reuptake transporter (SERT) by quantitative realtime PCR. After cisplatin administration, TPH2 expression increased significantly in the colon of rats compared to the control group on day 3 ( $p=0.008$, Figure 3(e)). The probiotic mixture greatly downregulated TPH1 $(p=0.042)$ and TPH2 $(p=0.013)$ in the colon of rats compared to the levels in the cisplatin-only rats (Figures $3(\mathrm{~d})$ and $3(\mathrm{e})$ ). The expression level of SERT in the colon of rats showed no significant differences (Figure 3(f)).
3.4. Regulation of Cisplatin-Induced Gut Dysbiosis in Rats by Probiotic Mixture Is Associated with Changes in the 5-HT Level. To reveal the effects of the probiotic mixture on the gut microbiota during cisplatin-induced mucositis, sequencing targeting the V3-V4 region of the $16 \mathrm{~S}$ rDNA was performed. Principal component analysis (PCA) revealed that the microbiota in the three groups were distinct from each other on day 3 , especially regarding principal component 1 (PC1; 51.2\%) (Figure 4(a)), indicating that cisplatin causes variation in the gut flora.

To further compare the gut microbiota composition among the three groups, histograms of the relative abundances were constructed, as shown in Figure 4(b) for phyla and Figure 4(c) for genera. The two most abundant bacterial phyla in rat were Firmicutes and Bacteroidetes. In the cisplatin-only rats, the relative abundance of Firmicutes increased (Figure S1A), while the relative abundance of Bacteroidetes decreased (Figure $\mathrm{S} 1 \mathrm{~B}$ ), and the ratio of Firmicutes to Bacteroidetes $(F / B)$ increased (Figure S1C), compared to the other two groups on day 3. Significant differences at the genus level were also observed (Figure 4(d)). Notably, the cisplatin-only rats had greater relative abundances of Enterobacteriaceae_other and Blautia on day 3 and Blautia and Clostridiaceae_other on day 6 but lower abundances of Lactobacillus (day 3) and Roseburia (day 6) than in the control group, suggesting that cisplatin closely affects the microbiota composition. Interestingly, in the PM group, Proteus, Fusobacterium, and Flexispira were clearly increased on day 3 compared to those in the cisplatin-only group. The relative abundances of Lactobacillus, Enterobacteriaceae other, Blautia, Roseburia, and Clostridiaceae_other decreased.

3.5. Probiotic Mixture Reduces Specific Bacteria That Promote Colonic 5-HT Biosynthesis In Vivo and Inhibit 5HT Secretion by EC (RIN-14B) Cells In Vitro. According to our finding that 5-HT levels were decreased in the colons of probiotic+cisplatin rats compared to the cisplatin-only rats, we therefore hypothesized that specific microbes are associated with affecting the host 5-HT pathways. Using Pearson's correlation analysis, bacteria that were significantly related to the colonic 5-HT variation were identified and plotted. We found that the genera Ruminococcaceae_other, Blautia, Ruminococcus, and Dorea were all significantly positively correlated with colonic 5-HT (Figure 5(a)). Relative quantification of selected 5-HT-associated bacterial groups was performed by quantitative real-time PCR. The results further revealed that the members of Clostridium clusters IV and XIVa (butyrate producers) were significantly increased in the cisplatin-only rats compared to the control rats. The PM group showed decreased abundances of Clostridium cluster IV and XIVa compared to the cisplatin-only group, especially on day $6(p=0.015$, Figure $5(\mathrm{~b}) ; p=0.03$, Figure $5(\mathrm{c})$ ).

To determine whether the protective effects of the probiotic mixture were directly associated with the inhibition of 5 -HT secretion by EC cells, we further subjected RIN-14B cells to no treatment (control), one of the four bacteria in the probiotic mixture or the mixture itself, cisplatin only, or cisplatin plus the probiotic mixture. We found that all four bacteria and the mixture itself significantly decreased 


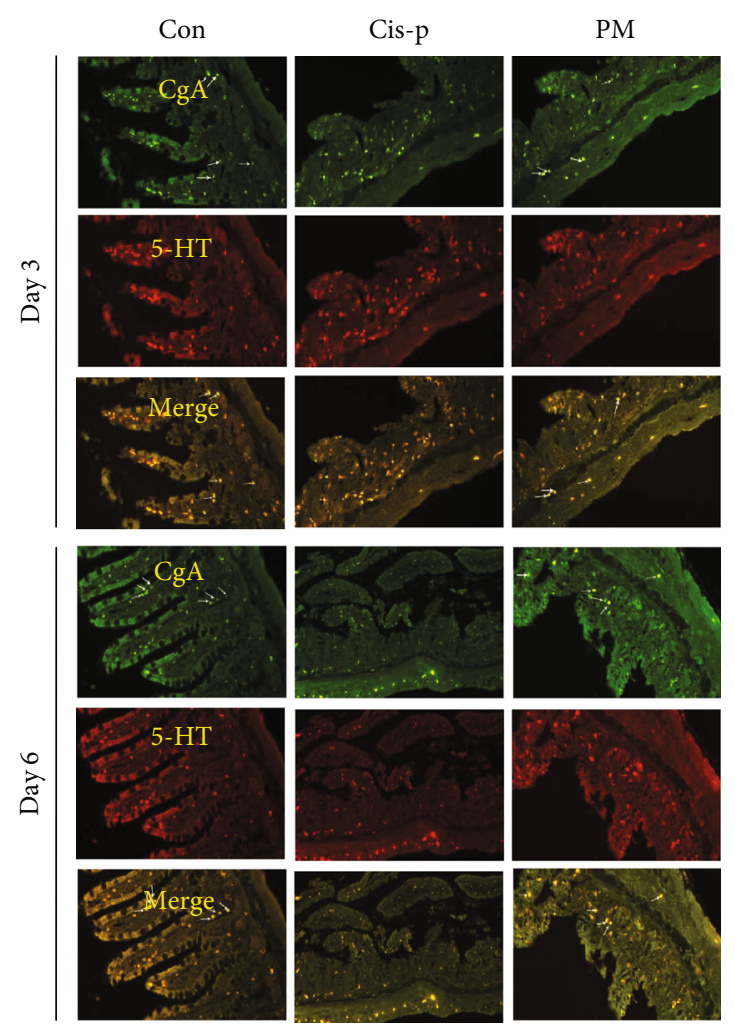

(a)

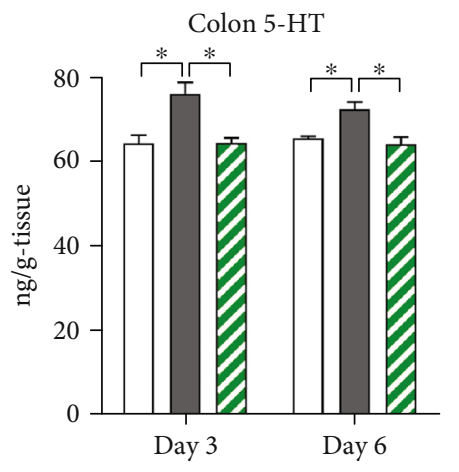

$\square$ Con

$\square$ Cis-p

एב PM

(b)
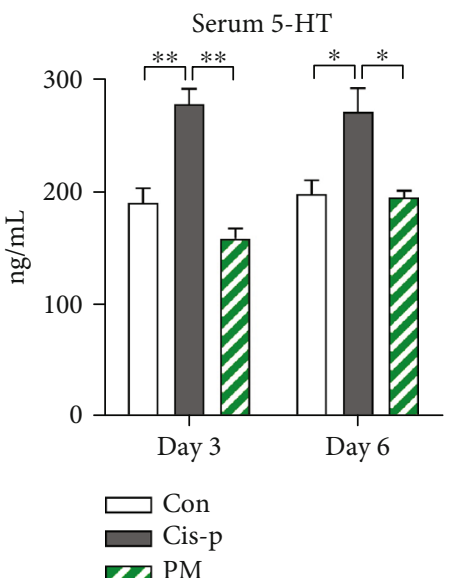

एव

(c)

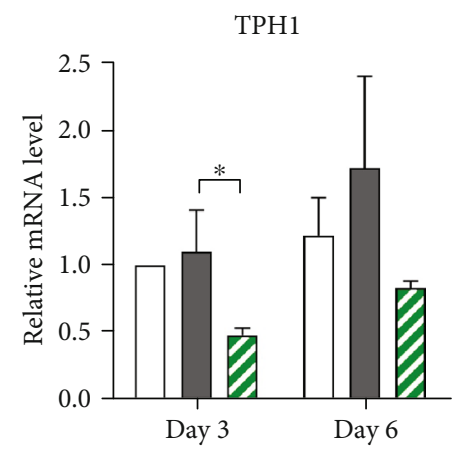

$\square$ Con

$\square$ Cis-p

एव PM

(d)

Figure 3: Continued. 


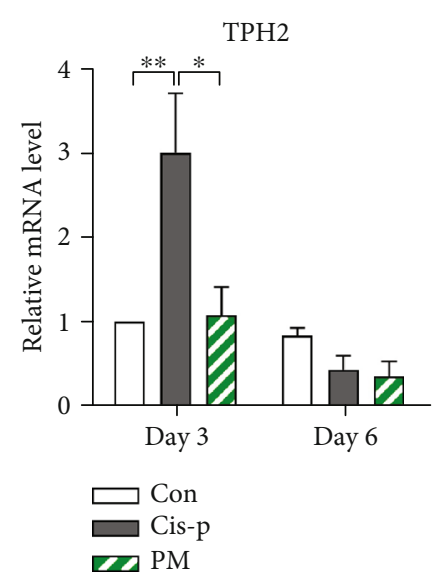

(e)

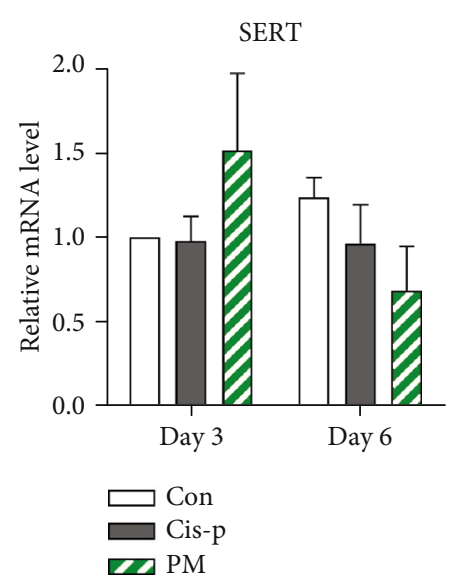

(f)

FIGURE 3: Effects of probiotic mixture on cisplatin-induced colonic 5-HT biosynthesis in rats. (a) Representative images of colons with chromogranin A (gastrointestinal endocrine cell marker, green) and 5-HT (red) staining. Levels of colonic (b) and serum (c) 5-HT. Colonic expression of TPH1 (d), TPH2 (e), and SERT (f) relative to $\beta$-actin. Data are normalized to expression levels in the control rats on day 3. Data are expressed as mean $\pm \operatorname{SEM}(n=4-5):{ }^{*} p<0.05$ and ${ }^{* *} p<0.01$.

5-HT secretion compared to the control cells (S. thermophilus, $p=0.003 ;$ L. casei, $p=0.002 ;$ L. acidophilus, $p=0.013 ; B$. brevis, $p=0.005$; PM, $p=0.009$; Figure 5(d)). Furthermore, the probiotic mixture significantly reduced cisplatininduced 5-HT overproduction (Cis-p vs. control, $p=0.002$; PM vs. Cis-p, $p=0.018$; Figure $5(d))$. This suggested that the probiotic mixture not only reduced 5-HT-associated bacteria but also had direct inhibitory effects on 5-HT biosynthesis in EC cells.

\section{Discussion}

In this study, the inhibitory effects of a probiotic mixture on cisplatin-induced mucositis and pica in rats were investigated. We found that the underlying mechanisms involved gut microbiota-induced alteration of gut-derived 5-HT biosynthesis.

Chemotherapy-induced mucositis and nausea/vomiting are common and major debilitating side effects of chemotherapy, which severely affects quality of life among cancer patients, even reducing their compliance with chemotherapy [30]. Cisplatin, a cytotoxic agent, is widely prescribed in chemotherapy regimens for various human cancers due to its affinity for DNA [31]. Cisplatin causes both acute and delayed GI tract disorders ( $<24$ and $>24$ hours after administration, respectively) [32]. Our results are consistent with those of prior studies in which cisplatin caused significant mucositis and pica in rats $[8,33-35]$. Another study revealed that treatment with the probiotic mixture used in our study (Bifidobacterium brevis DM8310, Lactobacillus acidophilus DM8302, Lactobacillus casei DM8121, and Streptococcus thermophilus DM8309) alleviates 5-fluorouracil-induced mucositis in rats [26]. Our study demonstrated that this probiotic mixture also attenuated cisplatin-induced mucositis and pica in rats, which involved reducing inflammation and oxidative stress. A clinical study found that treatment with $B$. breve ameliorated intestinal mucositis in pediatric cancer patients taking chemotherapy [36]. L. acidophilus and $L$. casei both significantly improved the inflammatory and functional aspects of 5-fluorouracil-induced intestinal mucositis in mice $[37,38]$. Furthermore, oral ingestion of S. thermophilus alleviated the symptoms of methotrexateinduced mucositis [39]. Thus, the probiotic mixture used in this study potentially improves GI function.

Intestinal barrier integrity is critical for intestinal function. Emerging data suggest that chemotherapy exerts detrimental effects on intestinal permeability, contributing to inflammation [40]. We detected histological abnormalities in the colon of mice treated with cisplatin, which were reversed by the probiotic mixture (Figure 2). In addition, compared to the cisplatin-only group, as indicated by the decreased endotoxin LPS in the serum. Although MUC-2 has been shown to have protective capacities, we found that it was upregulated during cisplatin-induced mucositis, especially on day 3 , and it reverted to a normal level by day 6 in the probiotic+cisplatin group. MUC-2 upregulation may be a counterreaction by the intestine to protect against mucositis [41], which was also observed in methotrexate-induced damage $[42,43]$. The mechanism may involve short-chain fatty acids (SCFAs) regulating prostaglandin production, thus stimulating MUC-2 expression in intestinal epithelial cells [44]. Our results also indicated that cisplatin-induced inflammation was attenuated by the probiotic mixture. The probiotic mixture inhibited cisplatin-induced generation of TNF- $\alpha$ and IL- 6 and promoted the secretion of IL-10. In the cisplatin-only rats, the increase in inflammatory cytokines may be attributable to the degradation of the epithelial barrier; furthermore, this may trigger dysbiosis and elicit secondary inflammation, ultimately resulting in intestinal mucositis [45]. Oxidative stress is also a critical component of cisplatin-induced intestinal injury [46]. There is evidence that some lactic acid bacteria (Bifidobacterium, Lactobacillus, Lactococcus, and Streptococcus thermophilus) exerted antioxidant activity [47]. Our results also revealed that the 

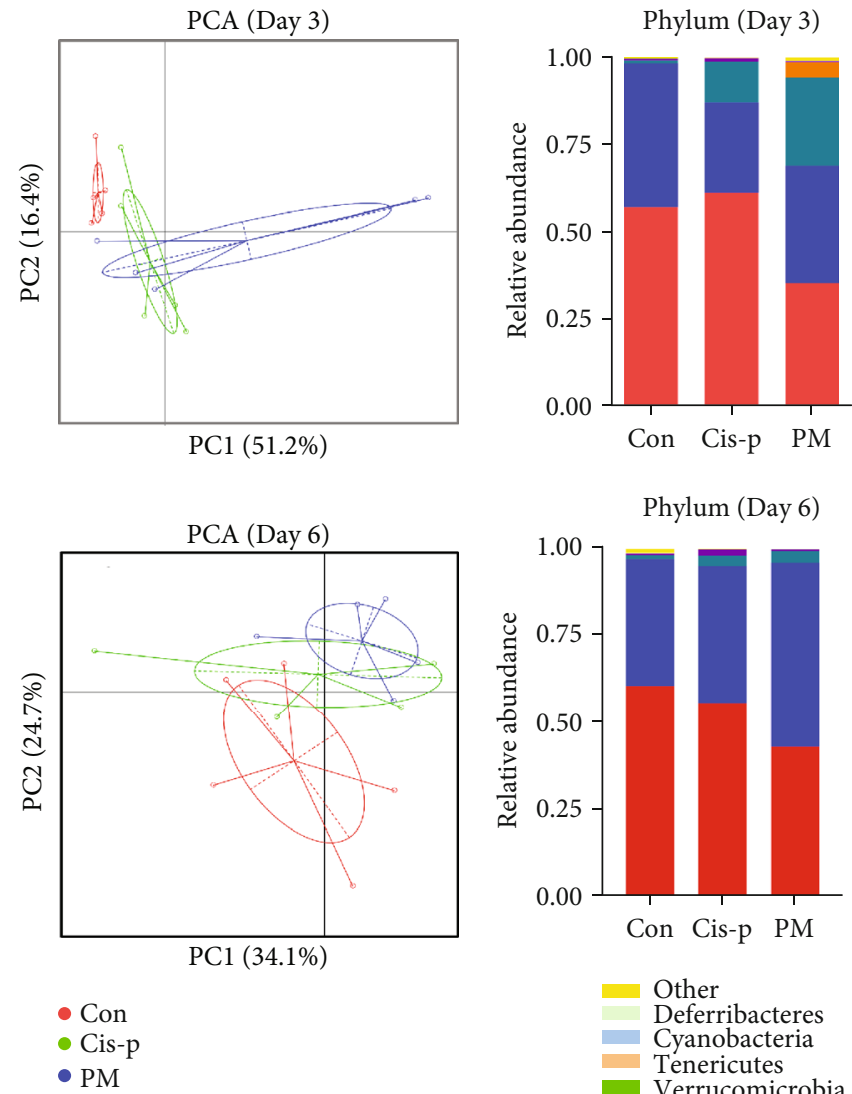

$$
\begin{aligned}
& \text { Other } \\
& \text { Deferribacteres } \\
& \text { Cyanobacteria } \\
& \text { Tenericutes } \\
& \text { Verrucomicrobia } \\
& \text { Actinobacteria } \\
& \text { Fusobacteria } \\
& \text { Proteobacteria } \\
& \text { Bacteroidetes }
\end{aligned}
$$

(a)

(b)

Figure 4: Continued. 

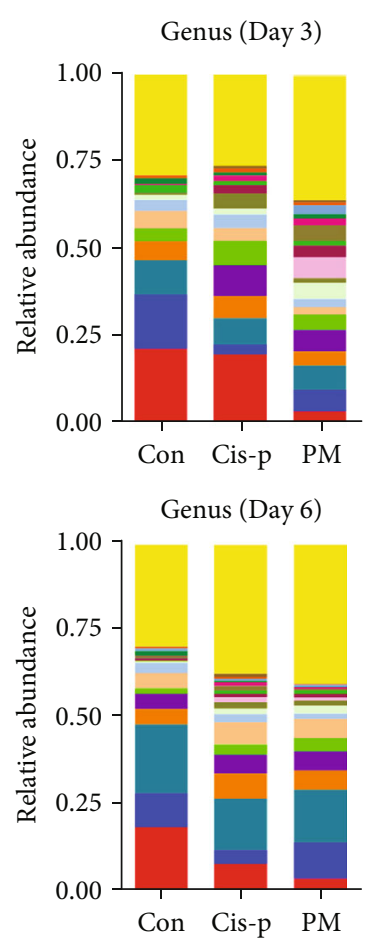

Other
Desulfovibrio
Clostridiaceae_other
Clostridium
Roseburia
Dorea
Ruminococcus
Enterobacteriaceae_other
Desulfovibrionaceae_other
Blautia
Phascolarctobacterium
Bacteroides
Ruminococcus
Oscillospira
Paraprevotella
Lachnospiraceae_other
Ruminococcaceae_other
Clostridiales_other
Prevotella
Lactobacillus

(c)
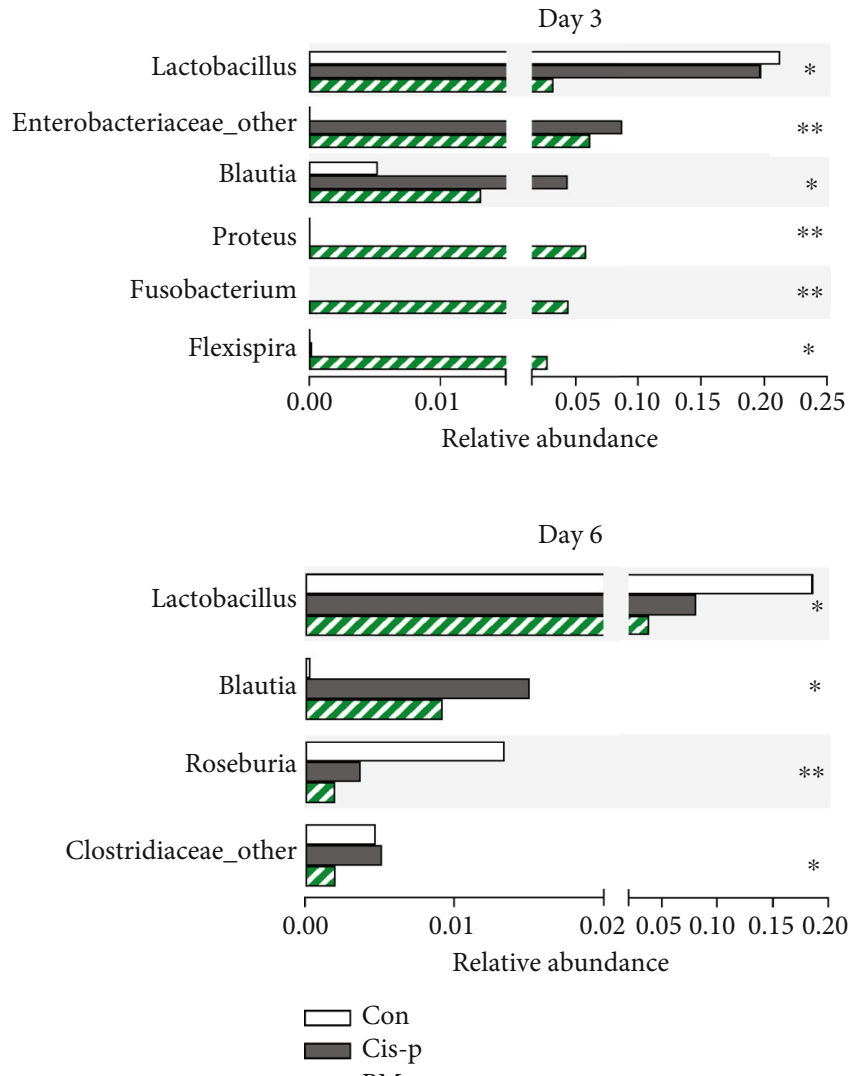

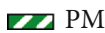

Figure 4: Probiotic mixture modulated intestinal bacterial composition in cisplatin-treated rats $(n=4-5)$. (a) Principal component analysis (PCA) among different samples. (b) Mean abundances in the gut microbiota at the phylum level. (c) Bar charts of the gut microbiota at the genus level. (d) Species differences among the three groups at the genus level. ${ }^{*} p<0.05$ and ${ }^{* *} p<0.01$.

probiotic mixture (Bifidobacterium, Lactobacillus, and Streptococcus thermophilus) reduced oxidative stress by decreasing MDA concentrations in both colon and ileum tissues. Thus, all the data suggested that the probiotic mixture has potentially anti-inflammatory and antioxidant activities.

It has been suggested that acute GI tract disorders involve 5-HT secretion from EC cells, which are a subset of enteroendocrine cells that reside within the intestinal mucosa [48]. A rate-limiting enzyme in 5-HT biosynthesis is $\mathrm{TPH}$, which is present in two isoforms (TPH1 and TPH2). TPH1 is primarily expressed in the EC cells of the gut, whereas TPH2 is expressed in serotonergic neurons [49]. TPH expression can be considered an indirect biomarker of 5-HT synthesis. 5-HT is inactivated by SERTmediated uptake into mucosal epithelial cells or enteric neurons [50]. Therefore, TPH and SERT are important for determining the 5-HT concentration and dynamics. A preliminary study revealed that cisplatin significantly increased TPH1 mRNA expression, but not TPH2 and SERT mRNA expression, in ileal tissue [51]. Another study demonstrated that cisplatin increased TPH1 and TPH2 mRNA expression in the ileum and medulla oblongata, respectively, indicating that cisplatin accelerates 5-HT synthesis through upregulating TPH, but has no effect on SERT [34]. We found that cisplatin significantly increased TPH1 and TPH2 levels in the colon but did not affect the SERT level. Thus, the increase in colonic TPH activity after cisplatin administration may be due to an increase in the number of EC cells and enteric neurons that express TPH1 and TPH2 mRNA. Furthermore, the probiotic mixture significantly inhibited the increase in 5-HT levels in the colon and serum, probably because it inhibited 5-HT synthesis in the colon (Figure 3). 


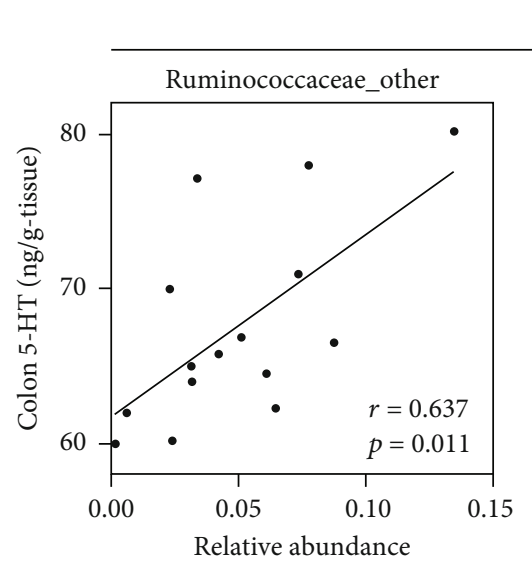

Day 3
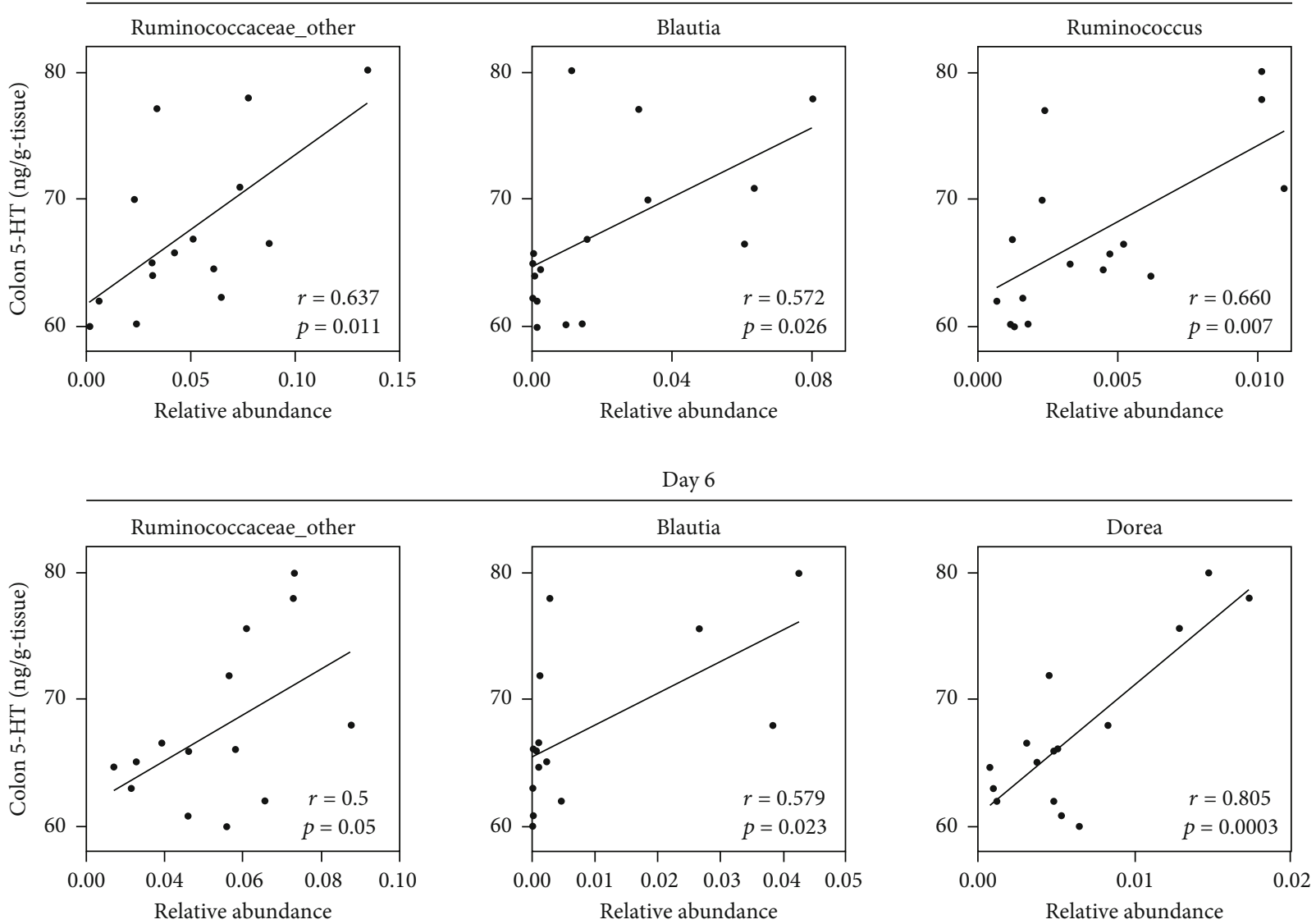

(a)
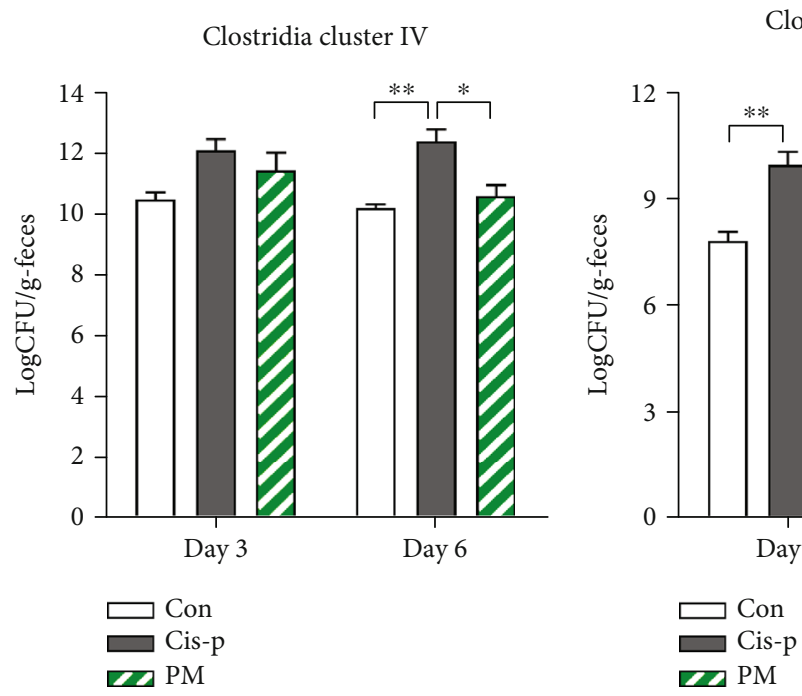

(b)

(c)

FIgUre 5: Continued. 


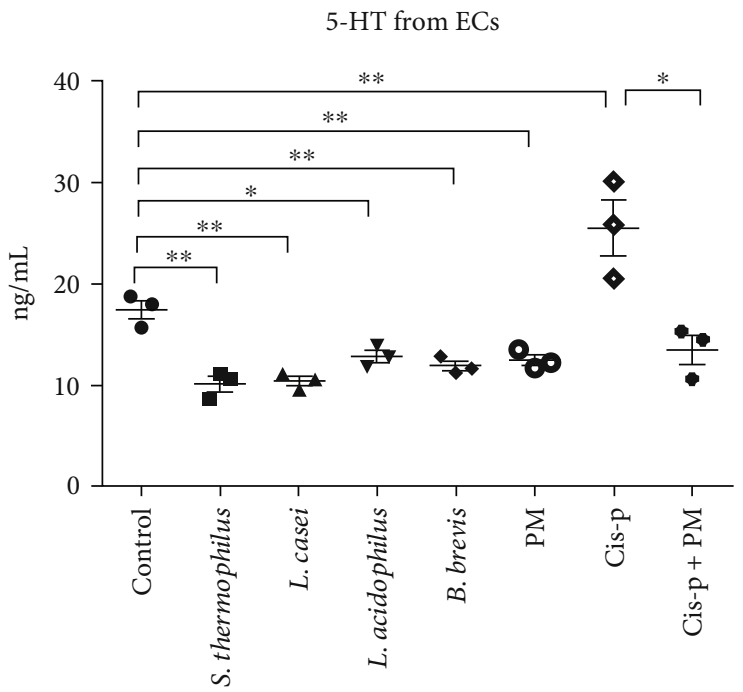

(d)

FIgURE 5: Probiotic mixture reduced 5-HT-associated bacteria and inhibited 5-HT secretion by RIN-14B cells. (a) Correlations between the abundances of differential species and colonic 5-HT level. Abundances of Clostridium cluster IV (b) and XIVa (c) in the feces of rats in different groups detected by quantitative PCR. Data are presented as the mean logarithm of bacterial colony-forming units (CFU) per gram of feces. (d) Levels of 5-HT released from RIN-14B cells after exposure to various bacteria or cisplatin. Data are expressed as mean \pm SEM $(n=3-5):{ }^{*} p<0.05,{ }^{* *} p<0.01$, and ${ }^{* * *} p<0.001$.

The gut microbiota plays an important role in promoting levels of colonic and serum 5-HT. Yano et al. [18] found that germ-free mice exhibited significantly decreased levels of colonic and serum 5-HT compared to specific pathogenfree (SPF) controls, and the study suggested that the microbiota regulated 5 -HT metabolism primarily by affecting host colonic EC cells. Gut dysbiosis is known to be involved in the pathogenesis of chemotherapy-induced mucositis, and restoring gut microbiota homeostasis may accelerate intestinal healing $[40,52,53]$. The probiotic mixture reversed cisplatin-induced gut dysbiosis-as indicated by the increased $F / B$ ratio $[54,55]$. We found that cisplatin treatment caused a significant increase in several gut bacterial taxa such as Enterobacteriaceae_other, Blautia, and Clostridiaceae_other, which were significantly restored by the probiotic mixture. Similar trends were observed in the abundances of Ruminococcaceae_other, Ruminococcus, and Dorea, although the differences were not significant. Notably, Ruminococcaceae_other, Blautia, Ruminococcus, and Dorea were all significantly positively correlated with colonic 5-HT (Figure 5). The abundances of Enterobacteriaceae and Blautia have been reported to be increased in chemotherapyinduced dysbiosis [56-58]. Consistent with our results, Ruminococcaceae and Ruminococcus have been reported to be positively correlated with 5-HT [59,60]. However, we also found that Proteus, Fusobacterium, and Flexispira, as putative gastrointestinal pathogens [61-63], were clearly increased in the PM group on day 3 compared with the other two groups (Figure 4(d)). We considered that the sample size in the Illumina Hiseq sequencing was not enough; further studies based on a larger number of samples should be conducted to confirm the results. Importantly, the gut microbiota compositions of the three groups are different on days 3 and 6 , which due to the gut epithelium have remarkable self-renewal capacity, and the intestinal microbiota also has the recovery ability to repair damaged mucosal barrier $[64,65]$. Furthermore, the probiotic mixture accelerated recovery of cisplatin-induced intestinal damage.

It is worth noting that SCFAs are well-known metabolites produced by the microbiota. They have been suggested to influence 5-HT secretion. The SCFAs' butyrate and acetate, which are produced in abundance by distal gut microbes in vivo, significantly affect enteric 5 -HT production by promoting TPH1 transcription [66]. Clostridia are anaerobic Firmicutes that produce a large array of metabolites. Moreover, Clostridium clusters IV and XIVa (also known as the Clostridium leptum and Clostridium coccoides groups, respectively) contain butyrate-producing species [67]. We therefore explored the link between Clostridium clusters IV and XIVa and cisplatin-induced intestinal injury. Riezzo et al. reported that a clinically relevant combination of irinotecan and 5-FU decreased Clostridium cluster IV and increased Clostridium cluster XIVa [68]. However, other studies showed that both an irinotecan regimen and 5-FU decreased the abundances of Clostridium cluster XIVa [26, 56]. Due to different drugs and different sampling times, our results demonstrated that cisplatin significantly increased members of Clostridium clusters IV and XIVa, which produce butyrate and thereby promote 5-HT secretion by EC cells. Therefore, we speculated that the probiotic mixture ameliorated cisplatin-induced mucositis and pica in rats partly by normalizing the dysbiosis-driven 5-HT overproduction. Additionally, probiotic bacterial species and probiotic supplements have a tendency to directly reduce colonic 5-HT $[69,70]$. We confirmed that the probiotic mixture also had direct inhibitory effects against 5-HT biosynthesis in EC cells in vitro. To sum up, the probiotic mixture supplementation in cisplatintreated rats reestablished the intestinal ecosystem, particularly 
the populations of probiotic bacteria, which in turn attenuated the increased 5-HT.

\section{Conclusion}

In this study, the protective effects of a probiotic mixture against cisplatin-induced mucositis and pica and the potential underlying mechanisms were evaluated. The probiotic mixture significantly ameliorated kaolin consumption, inflammation, oxidative stress, and the increased 5-HT concentrations caused by cisplatin. Furthermore, the probiotic mixture mitigated the gut dysbiosis and attenuated the altered metabolic profiles induced by cisplatin. The mechanism underlying the attenuation of cisplatin-induced intestinal injury may involve the probiotic mixture modulating the dysbiosis-driven 5-HT overproduction. The findings of this study indicate that the probiotic mixture may be useful for treating intestinal injury induced by cisplatin-based chemotherapy. However, more optimized study design and a larger sample size would benefit future studies. Further studies are required to fully understand the in-deep mechanisms of how the probiotic mixture supplementation ameliorates the cisplatin-induced mucositis and pica.

\section{Data Availability}

The raw data used to support the findings of this study will be made available by the authors, without undue reservation, to any qualified researcher.

\section{Ethical Approval}

All experimental protocols were approved by the Animal Ethical Committee of Dalian Medical University.

\section{Conflicts of Interest}

The authors have declared that no conflict of interest exists.

\section{Authors' Contributions}

YW performed the data analyses and wrote the manuscript. JW contributed to the conception of the study and obtained the funding. ZL and YL performed the experiments. QW and AW contributed significantly to analysis and manuscript preparation. XS helped perform the analysis with constructive discussions. JL contributed to the conception of the study and revised the manuscript. All authors read and approved the final manuscript.

\section{Acknowledgments}

This study was supported by the National Natural Science Foundation of China (82071911).

\section{Supplementary Materials}

Figure S1: composition analysis of gut microbiota at the phylum level $(n=4-5)$. (A) Proportion of Firmicutes; (B) proportion of Bacteroidetes; (C) the ratio of Firmicutes to Bacteroidetes. ${ }^{*} p<0.05$. (Supplementary Materials)

\section{References}

[1] N. Devarajan, R. Manjunathan, and S. K. Ganesan, “Tumor hypoxia: the major culprit behind cisplatin resistance in cancer patients," Critical Reviews in Oncology/Hematology, vol. 162, p. 103327, 2021.

[2] G. Babolmorad, A. Latif, I. K. Domingo et al., "Toll-like receptor 4 is activated by platinum and contributes to cisplatininduced ototoxicity," EMBO Reports, vol. 22, no. 5, article e51280, 2021.

[3] C. Rui, S. N. Shi, W. Ren et al., "The multitargeted kinase inhibitor KW-2449 ameliorates cisplatin-induced nephrotoxicity by targeting RIPK1-mediated necroptosis," Biochemical Pharmacology, vol. 188, p. 114542, 2021.

[4] S. Gong, Y. Feng, Y. Zeng et al., "Gut microbiota accelerates cisplatin-induced acute liver injury associated with robust inflammation and oxidative stress in mice," Journal of Translational Medicine, vol. 19, no. 1, p. 147, 2021.

[5] F. A. L. Barroso, L. C. L. de Jesus, C. P. de Castro et al., "Intake of Lactobacillus delbrueckii (pExu:hsp65) prevents the inflammation and the disorganization of the intestinal mucosa in a mouse model of mucositis," Microorganisms, vol. 9, no. 1, p. 107, 2021.

[6] Y. Zhang, B. Zhang, L. Dong, and P. Chang, "Potential of omega-3 polyunsaturated fatty acids in managing chemotherapy- or radiotherapy-related intestinal microbial dysbiosis," Advances in Nutrition, vol. 10, no. 1, pp. 133-147, 2019.

[7] L. S. Fideles, J. A. L. de Miranda, C. D. S. Martins et al., "Role of rutin in 5-fluorouracil-induced intestinal mucositis: prevention of histological damage and reduction of inflammation and oxidative stress," Molecules, vol. 25, no. 12, p. 2786, 2020.

[8] C. H. Wu, J. L. Ko, J. M. Liao et al., "D-Methionine alleviates cisplatin-induced mucositis by restoring the gut microbiota structure and improving intestinal inflammation," Therapeutic Advances in Medical Oncology, vol. 11, p. 175883591882102, 2019.

[9] P. J. Hesketh, "Chemotherapy-induced nausea and vomiting," The New England Journal of Medicine, vol. 358, no. 23, pp. 2482-2494, 2008.

[10] R. M. Navari and M. Aapro, "Antiemetic prophylaxis for chemotherapy-induced nausea and vomiting," The New England Journal of Medicine, vol. 374, no. 14, pp. 1356-1367, 2016.

[11] V. L. Batista, T. F. da Silva, L. C. L. de Jesus et al., "Probiotics, prebiotics, synbiotics, and paraprobiotics as a therapeutic alternative for intestinal mucositis," Frontiers in Microbiology, vol. 11, p. 544490, 2020.

[12] M. M. Wouters, S. J. Gibbons, J. L. Roeder et al., "Exogenous serotonin regulates proliferation of interstitial cells of Cajal in mouse jejunum through $5-\mathrm{HT}_{2 \mathrm{~B}}$ receptors," Gastroenterology, vol. 133, no. 3, pp. 897-906, 2007.

[13] R. Kon, N. Ikarashi, A. Hayakawa et al., "Morphine-induced constipation develops with increased aquaporin-3 expression in the colon via increased serotonin secretion," Toxicological Sciences, vol. 145, no. 2, pp. 337-347, 2015.

[14] Y. Dong, Y. Han, Z. Wang et al., "Role of serotonin on the intestinal mucosal immune response to stress-induced diarrhea in weaning mice," BMC Gastroenterology, vol. 17, no. 1, p. 82, 2017.

[15] A. Eliasen, K. Dalhoff, R. Mathiasen et al., "Pharmacogenetics of antiemetics for chemotherapy-induced nausea and vomiting: a 
systematic review and meta-analysis," Critical Reviews in Oncology/Hematology, vol. 149, p. 102939, 2020.

[16] R. Juza, P. Vlcek, E. Mezeiova, K. Musilek, O. Soukup, and J. Korabecny, "Recent advances with 5-HT3 modulators for neuropsychiatric and gastrointestinal disorders," Medicinal Research Reviews, vol. 40, no. 5, pp. 1593-1678, 2020.

[17] J. D. Matute, J. Duan, and R. S. Blumberg, "Microbial RNAs pressure piezol to respond," Cell, vol. 182, no. 3, pp. 542$544,2020$.

[18] J. M. Yano, K. Yu, G. P. Donaldson et al., "Indigenous bacteria from the gut microbiota regulate host serotonin biosynthesis," Cell, vol. 161, no. 2, pp. 264-276, 2015.

[19] H. Szőke, Z. Kovács, I. Bókkon et al., "Gut dysbiosis and serotonin: intestinal 5-HT as a ubiquitous membrane permeability regulator in host tissues, organs, and the brain," Reviews in the Neurosciences, vol. 31, no. 4, pp. 415-425, 2020.

[20] A. Perales-Puchalt, J. Perez-Sanz, K. K. Payne et al., "Frontline science: microbiota reconstitution restores intestinal integrity after cisplatin therapy," Journal of Leukocyte Biology, vol. 103, no. 5, pp. 799-805, 2018.

[21] X. Feng, Q. Cheng, Q. Meng, Y. Yang, and K. Nie, "Effects of ondansetron and [6]-gingerol on pica and gut microbiota in rats treated with cisplatin," Drug Design Development and Therapy, vol. 13, pp. 2633-2641, 2019.

[22] Y. T. Zou, J. Zhou, C. Y. Wu et al., "Protective effects of Poria cocos and its components against cisplatin-induced intestinal injury," Journal of Ethnopharmacology, vol. 269, p. 113722, 2021.

[23] C. C. Horn, B. A. Kimball, H. Wang et al., "Why can't rodents vomit? A comparative behavioral, anatomical, and physiological study," PLoS One, vol. 8, no. 4, article e60537, 2013.

[24] N. Takeda, S. Hasegawa, M. Morita, and T. Matsunaga, "Pica in rats is analogous to emesis: an animal model in emesis research," Pharmacology Biochemistry and Behavior, vol. 45, no. 4, pp. 817-821, 1993.

[25] Y. L. Liu, N. Malik, G. J. Sanger, M. I. Friedman, and P. L. Andrews, "Pica-a model of nausea? Species differences in response to cisplatin," Physiology \& Behavior, vol. 85, no. 3, pp. 271-277, 2005.

[26] Y. Tang, Y. Wu, Z. Huang et al., "Administration of probiotic mixture DM1 ameliorated 5-fluorouracil-induced intestinal mucositis and dysbiosis in rats," Nutrition, vol. 33, pp. 96104, 2017.

[27] M. Li, P. Liang, Z. Li et al., "Fecal microbiota transplantation and bacterial consortium transplantation have comparable effects on the re-establishment of mucosal barrier function in mice with intestinal dysbiosis," Frontiers in Microbiology, vol. 6, p. 692, 2015.

[28] Y. Y. Li, H. Liu, H. Qi et al., "Probiotic fermentation of Ganoderma lucidum fruiting body extracts promoted its immunostimulatory activity in mice with dexamethasoneinduced immunosuppression," Biomedicine \& Pharmacotherapy, vol. 141, p. 111909, 2021.

[29] M. Li, B. Wang, X. Sun et al., "Upregulation of intestinal barrier function in mice with DSS-induced colitis by a defined bacterial consortium is associated with expansion of IL-17A producing gamma delta $\mathrm{T}$ cells," Frontiers in Immunology, vol. 8, p. 824, 2017.

[30] L. Lohr, "Chemotherapy-induced nausea and vomiting," Cancer Journal, vol. 14, no. 2, pp. 85-93, 2008.

[31] M. R. Trendowski, O. El Charif, P. C. Dinh Jr., L. B. Travis, and M. E. Dolan, "Genetic and modifiable risk factors contributing to cisplatin-induced toxicities," Clinical Cancer Research, vol. 25, no. 4, pp. 1147-1155, 2019.

[32] H. Takeda, C. Sadakane, T. Hattori et al., "Rikkunshito, an herbal medicine, suppresses cisplatin-induced anorexia in rats via 5-HT2 receptor antagonism," Gastroenterology, vol. 134, no. 7, pp. 2004-2013, 2008.

[33] H. Yamamoto, K. Ishihara, Y. Takeda, W. Koizumi, and T. Ichikawa, "Changes in the mucus barrier during cisplatininduced intestinal mucositis in rats," BioMed Research International, vol. 2013, Article ID 276186, 8 pages, 2013.

[34] Q. Cheng, X. Feng, Q. Meng et al., “[6]-Gingerol ameliorates cisplatin-induced pica by regulating the TPH/MAOA/SERT/5-HT/5-HT3 receptor system in rats," Drug Design Development and Therapy., vol. 14, pp. 4085-4099, 2020.

[35] Y. Q. Li, Y. H. Yang, G. L. Zhang et al., "RNA-Seq reveals inflammatory mechanisms of Xiao-Ban-Xia-Tang decoction to ameliorate cisplatin-induced emesis in a rat pica model," Biomedicine \& Pharmacotherapy, vol. 131, p. 110699, 2020.

[36] Y. Yamashiro and S. Nagata, "Beneficial microbes for premature infants, and children with malignancy undergoing chemotherapy," Beneficial Microbes, vol. 1, no. 4, pp. 357-365, 2010.

[37] P. F. Justino, L. F. Melo, A. F. Nogueira et al., "Regulatory role of Lactobacillus acidophilus on inflammation and gastric dysmotility in intestinal mucositis induced by 5 -fluorouracil in mice," Cancer Chemotherapy and Pharmacology, vol. 75, no. 3, pp. 559-567, 2015.

[38] C. Y. Yeung, J. S. Chiang Chiau, M. L. Cheng et al., "Modulations of probiotics on gut microbiota in a 5-fluorouracilinduced mouse model of mucositis," Journal of Gastroenterology and Hepatology, vol. 35, no. 5, pp. 806-814, 2020.

[39] K. L. Tooley, G. S. Howarth, K. A. Lymn, A. Lawrence, and R. N. Butler, "Oral ingestion of streptococcus thermophilus diminishes severity of small intestinal mucositis in methotrexate treated rats," Cancer Biology \& Therapy, vol. 5, no. 6, pp. 593-600, 2006.

[40] S. Zhong, Z. Zhou, Y. Liang et al., "Targeting strategies for chemotherapy-induced peripheral neuropathy: does gut microbiota play a role?," Critical Reviews in Microbiology, vol. 45, no. 4, pp. 369-393, 2019.

[41] B. A. de Koning, M. van der Sluis, D. J. Lindenbergh-Kortleve et al., "Methotrexate-induced mucositis in mucin 2-deficient mice," Journal of Cellular Physiology, vol. 210, no. 1, pp. 144152, 2007.

[42] M. Verburg, I. B. Renes, H. P. Meijer et al., "Selective sparing of goblet cells and Paneth cells in the intestine of methotrexate-treated rats," The American Journal of PhysiologyGastrointestinal and Liver Physiology, vol. 279, no. 5, pp. G1037-G1047, 2000.

[43] M. Verburg, I. B. Renes, D. J. Van Nispen et al., "Specific responses in rat small intestinal epithelial mRNA expression and protein levels during chemotherapeutic damage and regeneration," Journal of Histochemistry \& Cytochemistry, vol. 50, no. 11, pp. 1525-1536, 2002.

[44] L. E. Willemsen, M. A. Koetsier, S. J. van Deventer, and E. A. van Tol, "Short chain fatty acids stimulate epithelial mucin 2 expression through differential effects on prostaglandin $\mathrm{E}(1)$ and $\mathrm{E}(2)$ production by intestinal myofibroblasts," Gut, vol. 52, no. 10, pp. 1442-1447, 2003.

[45] S. Kato, N. Hamouda, Y. Kano et al., "Probiotic Bifidobacterium bifidum G9-1 attenuates 5-fluorouracil-induced intestinal 
mucositis in mice via suppression of dysbiosis-related secondary inflammatory responses," Clinical and Experimental Pharmacology \& Physiology, vol. 44, no. 10, pp. 1017-1025, 2017.

[46] J. N. Hu, J. Y. Yang, S. Jiang et al., "Panax quinquefolium saponins protect against cisplatin evoked intestinal injury via ROS-mediated multiple mechanisms," Phytomedicine, vol. 82, p. 153446, 2021.

[47] A. Amaretti, M. di Nunzio, A. Pompei, S. Raimondi, M. Rossi, and A. Bordoni, "Antioxidant properties of potentially probiotic bacteria: in vitro and in vivo activities," Applied Microbiology and Biotechnology, vol. 97, no. 2, pp. 809-817, 2013.

[48] G. M. Mawe and J. M. Hoffman, "Serotonin signalling in the gut-functions, dysfunctions and therapeutic targets," Nature Reviews Gastroenterology \& Hepatology, vol. 10, no. 8, pp. 473-486, 2013.

[49] S. Matthes and M. Bader, "Peripheral serotonin synthesis as a new drug target," Trends in Pharmacological Sciences, vol. 39, no. 6, pp. 560-572, 2018.

[50] M. D. Gershon and J. Tack, "The serotonin signaling system: from basic understanding to drug development for functional GI disorders," Gastroenterology, vol. 132, no. 1, pp. 397-414, 2007.

[51] C. Ju, N. Hamaue, T. Machida et al., “Anti-inflammatory drugs ameliorate opposite enzymatic changes in ileal 5hydroxytryptamine metabolism in the delayed phase after cisplatin administration to rats," The European Journal of Pharmacology, vol. 589, no. 1-3, pp. 281-287, 2008.

[52] H. Wang, M. S. Geier, and G. S. Howarth, "Prebiotics: a potential treatment strategy for the chemotherapy-damaged gut?," Critical Reviews in Food Science and Nutrition, vol. 56, no. 6, pp. 946-956, 2016.

[53] E. Montassier, T. Gastinne, P. Vangay et al., "Chemotherapydriven dysbiosis in the intestinal microbiome," Alimentary Pharmacology \& Therapeutics, vol. 42, no. 5, pp. 515-528, 2015.

[54] X. B. Lin, L. A. Dieleman, A. Ketabi et al., "Irinotecan (CPT11) chemotherapy alters intestinal microbiota in tumour bearing rats," PLoS One, vol. 7, no. 7, article e39764, 2012.

[55] M. Fijlstra, M. Ferdous, A. M. Koning, E. H. Rings, H. J. Harmsen, and W. J. Tissing, "Substantial decreases in the number and diversity of microbiota during chemotherapy-induced gastrointestinal mucositis in a rat model," Supportive Care in Cancer, vol. 23, no. 6, pp. 1513-1522, 2015.

[56] J. Tong, X. Zhang, Y. Fan et al., "Changes of intestinal microbiota in ovarian cancer patients treated with surgery and chemotherapy," Cancer Management and Research, vol. Volume 12, pp. 8125-8135, 2020.

[57] Y. M. Kim, A. M. Snijders, C. J. Brislawn et al., "Light-stress influences the composition of the murine gut microbiome, memory function, and plasma metabolome," Frontiers in Molecular Biosciences, vol. 6, p. 108, 2019.

[58] A. T. Mudd, K. Berding, M. Wang, S. M. Donovan, and R. N. Dilger, "Serum cortisol mediates the relationship between fecal Ruminococcus and brain N-acetylaspartate in the young pig," Gut Microbes, vol. 8, no. 6, pp. 589-600, 2017.

[59] C. S. Reigstad, C. E. Salmonson, J. F. R. III et al., "Gut microbes promote colonic serotonin production through an effect of short-chain fatty acids on enterochromaffin cells," The FASEB Journal, vol. 29, no. 4, pp. 1395-1403, 2015.
[60] K. Atarashi, T. Tanoue, T. Shima et al., "Induction of colonic regulatory T cells by indigenous Clostridium species," Science, vol. 331, no. 6015, pp. 337-341, 2011.

[61] A. L. Hamilton, M. A. Kamm, S. C. Ng, and M. Morrison, "Proteus spp. as putative gastrointestinal pathogens," Clinical Microbiology Reviews, vol. 31, no. 3, article e00085, 2018.

[62] A. D. Kostic, D. Gevers, C. S. Pedamallu et al., "Genomic analysis identifies association of Fusobacterium with colorectal carcinoma," Genome Research, vol. 22, no. 2, pp. 292-298, 2012.

[63] M. Ying, Q. Yu, B. Zheng et al., "Cultured Cordyceps sinensis polysaccharides modulate intestinal mucosal immunity and gut microbiota in cyclophosphamide-treated mice," Carbohydrate Polymers, vol. 235, p. 115957, 2020.

[64] A. Gregorieff, Y. Liu, M. R. Inanlou, Y. Khomchuk, and J. L. Wrana, "Yap-dependent reprogramming of Lgr5(+) stem cells drives intestinal regeneration and cancer," Nature, vol. 526, no. 7575, pp. 715-718, 2015.

[65] Q. Hou, L. Ye, H. Liu et al., "Lactobacillus accelerates ISCs regeneration to protect the integrity of intestinal mucosa through activation of STAT3 signaling pathway induced by LPLs secretion of IL-22," Cell Death \& Differentiation, vol. 25, no. 9, pp. 1657-1670, 2018.

[66] Y. Touchefeu, E. Montassier, K. Nieman et al., "Systematic review: the role of the gut microbiota in chemotherapy- or radiation-induced gastrointestinal mucositis - current evidence and potential clinical applications," Alimentary Pharmacology \& Therapeutics, vol. 40, no. 5, pp. 409-421, 2014.

[67] H. Li, P. Wang, L. Huang, P. Li, and D. Zhang, "Effects of regulating gut microbiota on the serotonin metabolism in the chronic unpredictable mild stress rat model," Journal of Neurogastroenterology and Motility, vol. 31, no. 10, article e13677, 2019.

[68] G. Riezzo, G. Chimienti, A. Orlando, B. D'Attoma, C. Clemente, and F. Russo, "Effects of long-term administration of Lactobacillus reuteri DSM-17938 on circulating levels of 5-HT and BDNF in adults with functional constipation," Beneficial Microbes, vol. 10, no. 2, pp. 137-147, 2019.

[69] H. Yang, H. J. Kim, E. J. Hong, B. J. Pyun, B. S. Ko, and H. W. Lee, "Antidepressant effect of Tetragonia tetragonoides (Pall.) Kuntze extract on serotonin turnover," Evidence-based Complementary and Alternative Medicine, vol. 2019, Article ID 7312842, 7 pages, 2019.

[70] T. Matsuki, K. Watanabe, J. Fujimoto, T. Takada, and R. Tanaka, "Use of $16 \mathrm{~S}$ rRNA gene-targeted group-specific primers for real-time PCR analysis of predominant bacteria in human feces," Applied and Environmental Microbiology, vol. 70, no. 12, pp. 7220-7228, 2004.

[71] M. I. Van Dyke and A. J. McCarthy, "Molecular biological detection and characterization of Clostridium populations in municipal landfill sites," Applied and Environmental Microbiology, vol. 68, no. 4, pp. 2049-2053, 2002.

[72] H. Yang, R. Cai, Z. Kong et al., "Teasaponin ameliorates murine colitis by regulating gut microbiota and suppressing the immune system response," Frontiers of Medicine, vol. 7, p. $584369,2020$.

[73] M. Mulder, D. Radjabzadeh, J. C. Kiefte-de Jong et al., "Longterm effects of antimicrobial drugs on the composition of the human gut microbiota," Gut Microbes, vol. 12, no. 1, article 1795492, 2020. 